\title{
Design and Simulation of Air to Air Missile Homing System
}

\author{
Rahmat Alfi Duhri ${ }^{1 *}$, Rianto Adhy Sasongko ${ }^{1 *}$, and Yayom Dwi Laksmana ${ }^{2 *}$
}

\begin{abstract}
This paper will talk about AIM 120 AMRAAM missile guidance for pursuing a moving target. The missile guidance system itself consists of missile dynamics, control system, seeker, and guidance methods. For general purpose, the missile dynamics approach will use non-linear equation of motions. The control surface that will be discussed follow the rule BTT (Bank-to-Turn) and control system that will be used is PID control system that widely used for control design. Lastly, the guidance method that will be studied here is proportional navigation and constant bearing course approach. The simulation will be conducted using MATLAB Simulink. The Simulink model consist of target dynamics, and guidance system. From the result of simulation, it will be shown that the missile can pursue its target quite well. Hence, the simulation system can be used well for preliminary design purpose.
\end{abstract}

Keywords-Homing System, Control System, Proportional Navigation, Missile Dynamics, Seeker, Bank-to-Turn, Constant Bearing Course.

\section{INTRODUCTION}

$\mathbf{T}$ HE security systems of a country nowadays can be represented by the advanced technologies used for defending against intruders. One of those technologies is missile homing system of missile technology. With this technology, the intended target can be shot without any errors caused by human. There are a lot of limitations that can make this human factors become major effects of missing target such as anxiety, nervous, panic, low sight, etc. Hence, it is important to make the missile can pursue its target by itself.

The homing system basically use the concept of tracking and processing the trace of target and take it as commands to the missile for doing certain maneuver. In other words, missile's sensor will receive and interpret the locations and direction of target. After that, the computer or microprocessor that already had those directions will use the locations information as the reference for giving command to the missile through the control surfaces of the missile. The missile will follow this command, following certain path, and eventually it can hit the target. The exact algorithm of this homing system can be seen in the Fig. 1

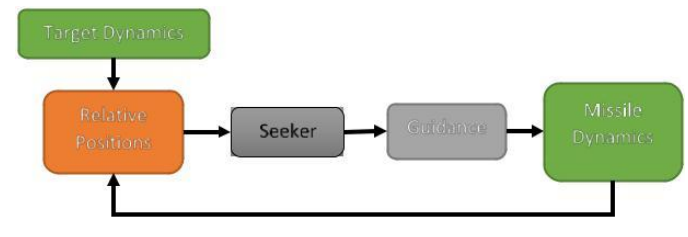

Fig. 1. Algorithm of Missile Guidance System

\footnotetext{
${ }^{1}$ The Aerospace Department, Bandung Institute of Technology, Jalan Ganesha No. 10, Bandung.

${ }^{2}$ Garuda Maintenance Facility AeroAsia, Soekarno Hatta International
} Airport, Cengkareng PO. BOX 1303 BUSH 19100

"Correspondence to the authors' email: alfiduhri@gmail.com, sasongko@ae.itb.ac.id, yayomdwi41@ gmail.com
The guidance can be achieved by a lot of methods and approaches, but in this paper, the methods that will be used is proportional navigation method, that will be explained in the other part of this paper. As for approach, in this paper, it means the routine that must be followed by the missile in order to pursue the target until it hits the target. In this paper, constant bearing approach will be used. This approach basically take advantage of parallelism of two objects. When two objects make a certain parallelism, as both of them move, they will meet at a certain point. For illustration, Fig. 2. below can explain how constant bearing course work.

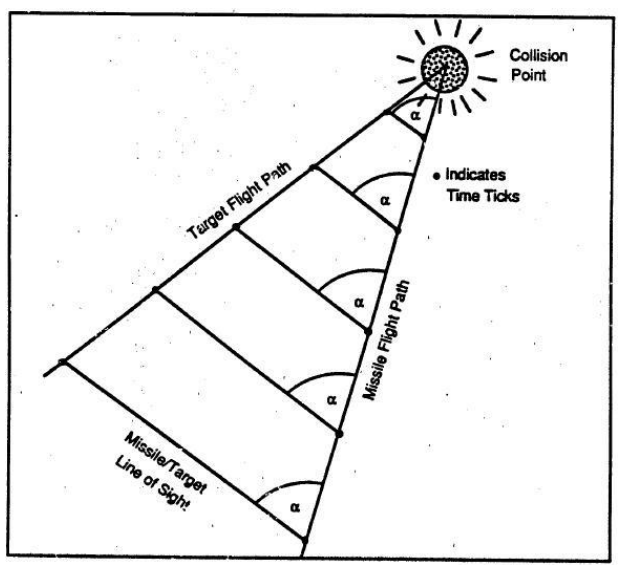

Fig. 2. Constant Bearing Course Scheme [3]

The model that is used in this paper is missile AIM 120 AMRAAM (Advanced Medium Range Air-to-Air Missile), as shown in Fig. 3. The name suggest that the missile operate from flying aircraft to shot the flying object.

The overview of the work in the paper have been written in this chapter. In the chapter II, the paper will talk about homing system, its components, and what is the important part of homing system. The next paragraph will explain the equations of motion for both missile and target, and control system that will be used in supporting guidance of missile. In the IV paragraph, the simulation and the result will be shown and discussed. Finally, the last one is Conclusion of this paper.

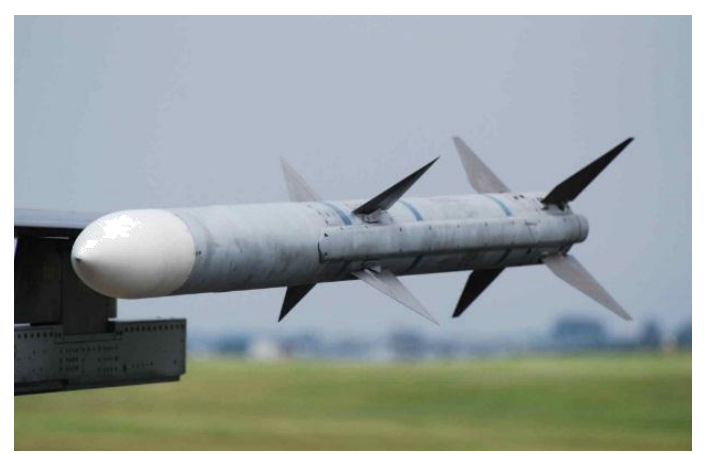

Fig. 3. AIM 120 AMRAAM [7] 


\section{II.HOMING SYSTEMS}

Homing system can be divided into three components, those are seeker system, navigation system, and autopilot system or guidance law. Homing system also need other parameters that correspond with aircraft dynamics, they are called Engagement geometries.

\section{A. Proportional Navigation}

Proportional Navigation is commonly used method for designing missile guidance. The basic idea of this method is the acceleration command generated in the missile is proportional to the line of sight (LOS) rate. It can be shown in Fig. 4.

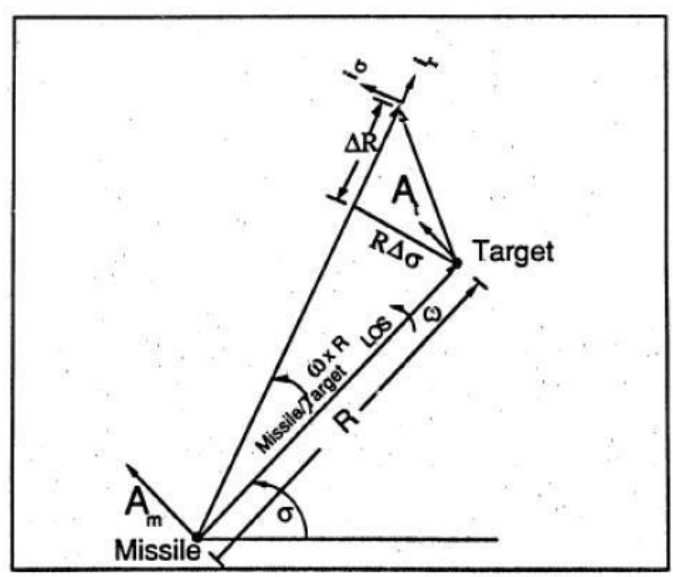

Fig. 4. Proportional Navigation System Scheme [3]

The basic idea of this navigation is to maintain missile LOS rate become zero and the missile will have constant bearing course with the target until it hit the target. It can be achieved by giving acceleration command to the missile. The equation of Proportional Navigation can be written as [3]:

$$
\gamma=N \sigma^{\circ}
$$

where, $\dot{\gamma}$ is rate of change of missile heading, $N$ is navigation ratio, $\sigma^{*}$ is line of sight rate.

Navigation Ratio represents the gain that will be processed as command to the missile. The more the value, the missile will be more aggressive with smaller LOS rate. In the other hand, the less the value, the missile will less responsive toward the change of LOS.

\section{B. Seeker System}

Seeker is the components of homing for censoring the location of the target. The seeker can detect and follow the movements of the target by sensing the unique characteristics

$$
u_{\text {gui }}=\left[\begin{array}{c}
\dot{\beta}_{\text {pitch }} \\
\dot{\beta}_{\text {yaw }}
\end{array}\right] \quad x_{\text {gui }}=\left[\begin{array}{c}
\gamma_{m_{\text {pitch }}} \\
\gamma_{m_{\text {yaw }}}
\end{array}\right]
$$

$$
\dot{x}_{g u i}=A x_{g u i}+B u_{g u i}
$$

of the target, such as radiation or reflection of energy by the target.

In the guidance system seeker will sense the target with certain error. This can be caused by the movement of seeker head sometimes is slower than the movement of target. Considering that condition, the seeker equation will become the state space as below [3]:

$$
\dot{x_{s h}}=A x_{s h}+B u_{s h}
$$

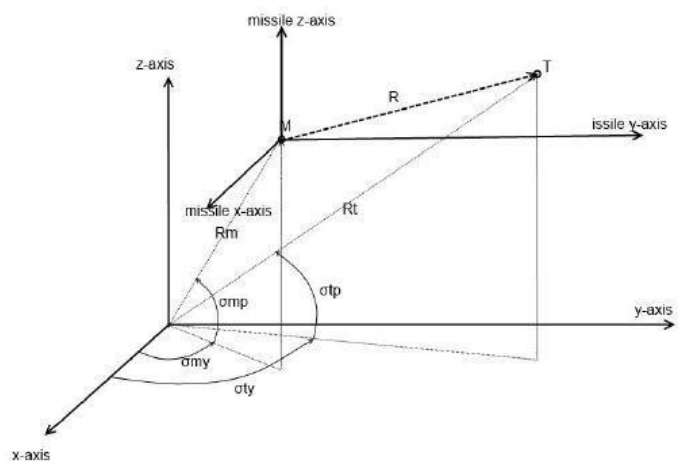

Fig. 5. Seeker Sensing Illustration [4]

where [3],

$$
\begin{gathered}
x_{\text {sh }}=\left[\begin{array}{llll}
\beta_{\text {pitch }} & \dot{\beta}_{\text {pitch }} & \beta_{\text {yaw }} & \dot{\beta}_{\text {yaw }}
\end{array}\right], u_{\text {sh }}=\left[\begin{array}{c}
\sigma_{\text {pitch }} \\
\sigma_{\text {yaw }}
\end{array}\right] \\
A=\left[\begin{array}{cccc}
0 & 1 & 0 & 0 \\
-k_{1} & -k_{2} & 0 & 0 \\
0 & 0 & 0 & 1 \\
0 & 0 & -k_{1} & -k_{2}
\end{array}\right] B=\left[\begin{array}{cc}
0 & 0 \\
k_{1} & 0 \\
0 & 0 \\
0 & k_{1}
\end{array}\right]
\end{gathered}
$$

with, $\beta_{\text {pitch }}$ is seeker output LOS in pitch direction, $\beta_{y a w}$ is seeker output LOS in yaw direction, $\sigma_{\text {pitch }}$ is seeker input LOS in pitch direction, $\sigma_{\text {yaw }}$ is seeker input LOS in yaw direction, $k_{1}$ is seeker ratio $=100, k_{2}$ is seeker ratio $=20$. In the equations of seeker that have been mentioned before, seeker ratio determine the accuracy of seeker and every seeker have this value. For more convenience we will choose the value as stated above.

\section{Guidance Law}

In part $A$ of Chapter II, the equation of proportional navigation have been stated clearly. In order to implement it into the guidance system, the guidance law must be derived. The guidance law within the missile system determines the best trajectory for the missile based on missile position, target position, missile capability, and desired objectives [3]. The following equation is the guidance law in term of state space [3]. 


$$
A=\left[\begin{array}{cc}
-k & 0 \\
0 & -k
\end{array}\right] \quad B=\left[\begin{array}{cc}
N & 0 \\
0 & N
\end{array}\right]
$$

After that, the heading of the missile, will be applied in equation below [3].

$$
\begin{gathered}
V_{m_{\text {pitch }}}=V_{m} \cos \left(\gamma_{m_{\text {yaw }}}-\sigma_{\text {yaw }}\right) \\
V_{m_{\text {yaw }}}=V_{m} \cos \left(\gamma_{m_{\text {pitch }}}\right) \\
A_{m_{\text {pitch }}}=V_{m_{\text {pitch }}} \cos \left(\dot{\gamma}_{m_{\text {pitch }}}\right) \\
A_{m_{\text {yaw }}}=V_{m_{\text {yaw }}} \cos \left(\dot{\gamma}_{m_{\text {yaw }}}\right)
\end{gathered}
$$

\section{Engagement Geometry}

The engagement geometry represent the flight paparameters of both missile and target which are needed for guidance system simulation.

1) Line of Sight (LOS): Line of sight is the angle between the missile and the target, see Fig.6.

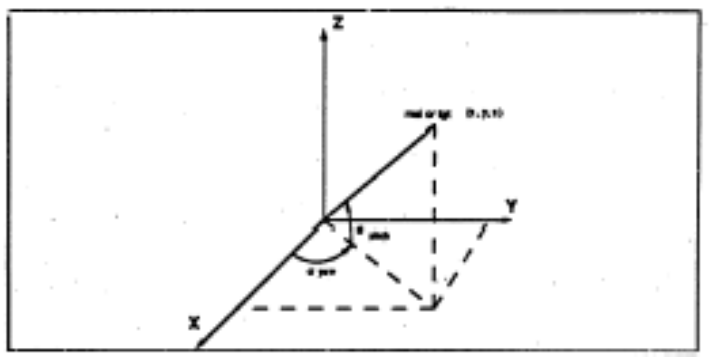

Fig.6. Line of Sight Illustration

The equation of LOS can be written below [3]

$$
\begin{gathered}
\sigma_{\text {pitch }}=\tan ^{-1}\left[\frac{z_{t}-z_{m}}{\sqrt{\left(x_{t}-x_{m}\right)^{2}+\left(y_{t}-y_{m}\right)^{2}}}\right] \\
\sigma_{y a w}=\tan ^{-1}\left[\frac{z_{t}-z_{m}}{x_{t}-x_{m}}\right]
\end{gathered}
$$

2) Flight Path Angels : Flight path angle represent the attitude of the missile and the target along their trajectory, see Fig.7.

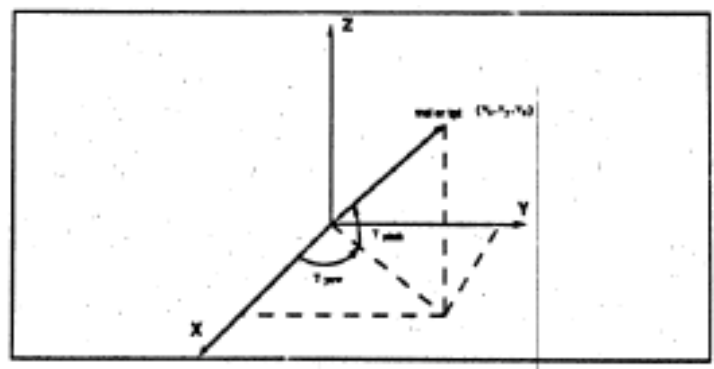

Fig.7. Flight Path Angles Illustration

The equation of flight path angels can be written below [3]

$$
\gamma_{\text {pitch }}=\tan ^{-1}\left[\frac{v-V_{m_{z}}}{\left(v_{t_{x}}-V_{m_{x}}\right)^{2}+\left(V_{t_{y}}-V_{m_{y}}\right)^{2}}\right]
$$

$$
\gamma_{y a w}=\tan ^{-1}\left[\frac{V_{t_{z}}-V_{m_{z}}}{v_{t_{x}}-V_{m_{x}}}\right]
$$

3) Velocity and Acceleration: Velocity and Acceleration is important factors here because both of them determine the trajectory and the movement of missile and target. In this section, the one that will be focused is missile acceleration and velocity because in the guidance system, the acceleration and velocity of missile have direct cause to the movement change [3].

$$
\begin{aligned}
& V_{m}=\sqrt{V_{m_{x}}+V_{m_{y}}+V_{m_{z}}} \\
& A_{m}=\sqrt{A_{m_{x}}+A_{m_{y}}+A_{m_{z}}}
\end{aligned}
$$

In the yaw direction, the acceleration equation can be represented below [3].

$$
\begin{gathered}
\ddot{x}_{m_{\text {pitch }}}=-\left(A_{m_{\text {pitch }}} \sin \sigma_{\text {pitch }}\right) \cos \sigma_{\text {pitch }} \\
\ddot{y}_{m_{\text {pitch }}}=-\left(A_{m_{\text {pitch }}} \sin \sigma_{\text {pitch }}\right) \cos \sigma_{\text {yaw }} \\
\ddot{z}_{m_{\text {pitch }}}=-\left(A_{m_{\text {pitch }}} \sin \sigma_{\text {pitch }}\right)
\end{gathered}
$$

In the yaw direction, the acceleration equation can be represented below [3].

$$
\begin{aligned}
& \ddot{x}_{m_{\text {yaw }}}=-A_{m_{\text {yaw }}} \sin \sigma_{\text {yaw }} \\
& \ddot{y}_{m_{\text {yaw }}}=-A_{m_{\text {yaw }}} \operatorname{Cos} \sigma_{\text {yaw }}
\end{aligned}
$$

Both acceleration will become the basis for deriving tracking controller of missile. The total acceleration that the missile will have can be shown below [3].

$$
\begin{aligned}
& \ddot{x}_{m}=\ddot{x}_{m_{\text {pitch }}}+\ddot{x}_{m_{\text {yaw }}} \\
& \ddot{y}_{m}=\ddot{y}_{m_{\text {pitch }}}+\ddot{y}_{m_{\text {yaw }}} \\
& \ddot{z}_{m}=\ddot{z}_{m_{\text {pitch }}}+\ddot{x}_{m_{\text {yaw }}}
\end{aligned}
$$

\section{DYNAMIC EQUATIONS AND CONTROL SYSTEM}

\section{A. Missile Dyanamic Equation}

Missile dyanamic equation that will be used here is nonlinear dynamic equations of motion. The equation of motion consist of tranlational equations (equation 22), rotational equations (equation 23), and kinematic equations of motion. The non-linear equation is chosen because it can generalize the system in any flight conditions.

$$
\begin{aligned}
& \overrightarrow{\mathrm{F}}_{\mathrm{A}}+\overrightarrow{\mathrm{W}}_{\mathrm{b}}+\overrightarrow{\mathrm{F}}_{\mathrm{p}}=\mathrm{m}\left(\frac{\partial \overrightarrow{\mathrm{v}}}{\partial \mathrm{t}}+\vec{\omega} \times \overrightarrow{\mathrm{V}}\right) \\
& \overrightarrow{\mathrm{M}}_{\mathrm{A}}+\overrightarrow{\mathrm{M}}_{\mathrm{p}}=\left(\mathrm{I} \frac{\partial \vec{\omega}}{\partial \mathrm{t}}+\vec{\omega} \times(\mathrm{I} \cdot \vec{\omega})\right)
\end{aligned}
$$

For modelling dynamic equations of motion, there are aerodynamics forces and moments. They are calculated using Missile DATCOM [1]. Instead of using experimental data, Missile DATCOM used because it is easy to use, and have good approximation result. 


\section{B. Target Dynamic equations}

The target dynamic equation of motion just consist of translational equations. For this paper, this is enough because the main purpose of this paper is not studying the performance of guidance, but for studying how the guidance system work for non-linear missile system. Lastly, the equation of motions for target can be shown belw [3].

Where,

$$
\dot{x}_{t}=A x_{t}+B u_{t}
$$

$$
\begin{array}{ccc}
x_{t}=\left[\begin{array}{llllll}
x_{t} & \dot{x}_{t} & y_{t} & \dot{y}_{t} & z_{t} & \dot{z}_{t}
\end{array}\right]^{T}, u_{t}=\left[\begin{array}{lll}
\ddot{x}_{t} & \ddot{y}_{t} & \ddot{z}_{t}
\end{array}\right]^{T} \\
A=\left[\begin{array}{llllll}
0 & 1 & 0 & 0 & 0 & 0 \\
0 & 0 & 0 & 0 & 0 & 0 \\
0 & 0 & 0 & 1 & 0 & 0 \\
0 & 0 & 0 & 0 & 0 & 0 \\
0 & 0 & 0 & 0 & 0 & 1 \\
0 & 0 & 0 & 0 & 0 & 0
\end{array}\right], \quad B=\left[\begin{array}{lll}
0 & 0 & 0 \\
1 & 0 & 0 \\
0 & 0 & 0 \\
0 & 1 & 0 \\
0 & 0 & 0 \\
0 & 0 & 1
\end{array}\right]
\end{array}
$$

In the state space above, the input of the equations is the acceleration of the state. That state can be used for moving object both with acceleration and without acceleration.

\section{Control System}

1) Tracking Controller: Tracking controller is close loop feed back system with the purpose to derive the system to track the input that can be shown by the exact tendency of certain output to follow the input. There are a lot of methods to implement tracking controller into the system. One of the them is using PID controller that will be used in this paper.

2) Bank-to-Turn : Bank-to-turn is the type of control surface in missile which can only the missile to roll and pitch. It is because the missile just only have a pair of symmetric control surface which can generate pitching moment and a pair of asymmetric control surface that can make the missile able to roll. For tracking controller in homing system, the pitch error can calculated using the equation below [2].

$$
\varphi=\tan ^{-1} \frac{a_{y}}{a_{\mathrm{z}}}
$$

where, $a_{y}$ and $a_{z}$ are both component of missile acceleration.

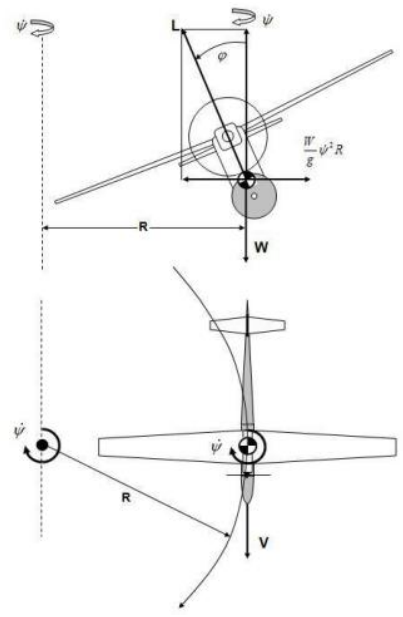

Fig. 8. Bank-to-Turn Manuever

3) PID Controller: PID stands for Proportional Integral Derivative. As the name of its abbreviation, PID itself consists of Proportional gain, Integral gain, and Derivative gain. Proportional gain have a function to amplify the control but does not change the dynamics of control. Integral gain can improve and at the same time also can nullify steady state response. The last one, Derivative gain can improve transient response by improving the error rate that will happen.

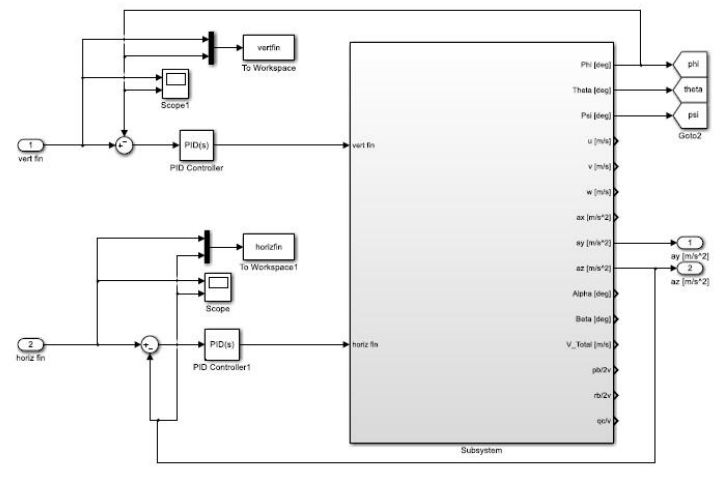

Fig. 9. PID Controller for Bank-to-Turn

\section{SIMULATION AND RESULT}

The simulation of missile guidance was conducted in MATLAB Simulink. The result of several case can be seen in this chapter.

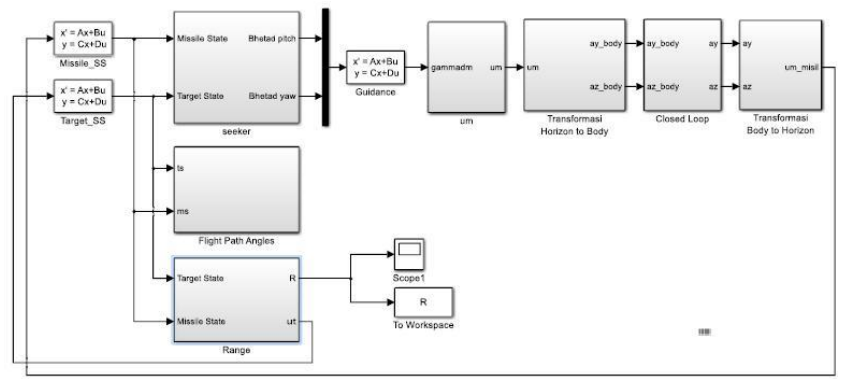

Fig. 10. System Guidance Simulation Model

The model above is used for simulation of missile guidance in pursuing the target. For example, below is the initial condition of a case.

Tabel 1 Initial Condition Misil Dan Target

\begin{tabular}{lll}
\hline \hline & Missile & Target \\
\hline$X(\mathrm{~m})$ & 0 & 10000 \\
$\mathrm{Y}(\mathrm{m})$ & 0 & 0 \\
$\mathrm{Z}(\mathrm{m})$ & 4572 & 4572 \\
$\mathrm{Vx}(\mathrm{m} / \mathrm{s})$ & 1020 & 0 \\
$\mathrm{Vy}(\mathrm{m} / \mathrm{s})$ & 0 & 100 \\
$\mathrm{Vz}(\mathrm{m} / \mathrm{s})$ & 0 & 100 \\
$\mathrm{Ax}\left(\mathrm{m} / \mathrm{s}^{\wedge} 2\right)$ & 0 & 100 \\
$\mathrm{Ay}\left(\mathrm{m} / \mathrm{s}^{\wedge} 2\right)$ & 0 & 0 \\
$\mathrm{Az}\left(\mathrm{m} / \mathrm{s}^{\wedge} 2\right)$ & 0 & 0 \\
\hline
\end{tabular}

The result of the simulation can be shown below. 


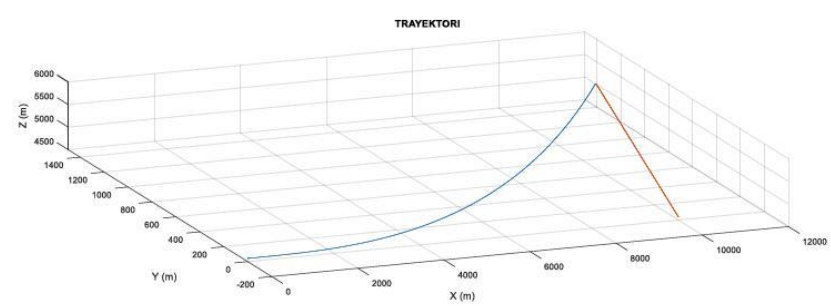

Fig. 11. Trajectory of Missile (Blue) and Target (Red)

The above figure show the trajectory of missile and target from initial position until the missile hit the target. From the trajectory, we know that the missile finally can meet the target at one point. Below is the range between the missile and the target that can prove that statement.

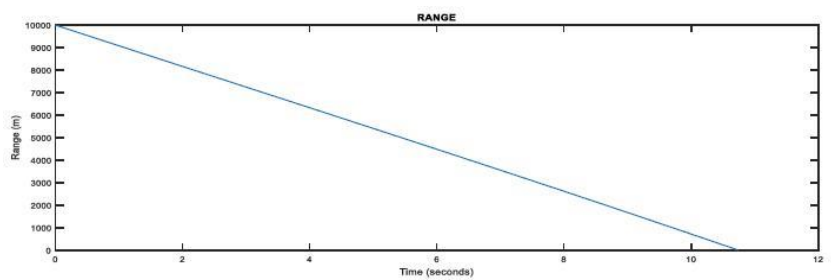

Fig. 12. Range between Missile and Target

As for Line of Sight of the missile, the missile continuously undergoes change of Line of Sight with small changing. Ideally, there will be no change in Line of Sight. We know that there are limitations in seeker, guidance system, numeric modelling, etc. can influence the result of simulation. Thus, this is what happen. There will be small change that does not affect the performance of guidance.

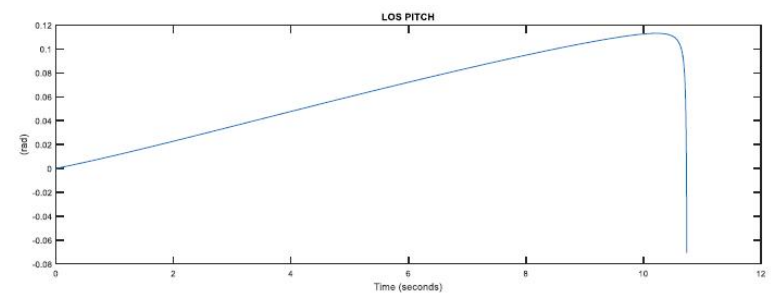

Fig. 13. LOS in Pitch Direction between Missile and Target

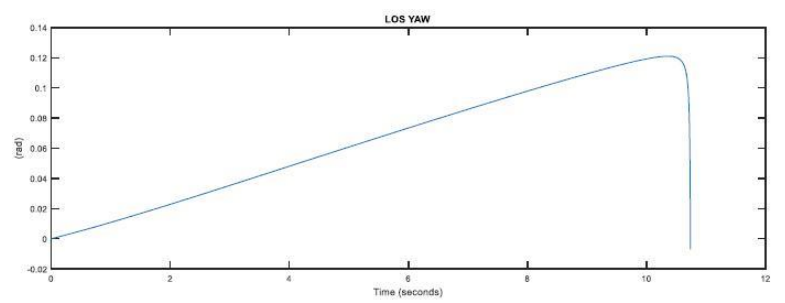

Fig. 14. LOS in Yaw Direction between Missile and Target

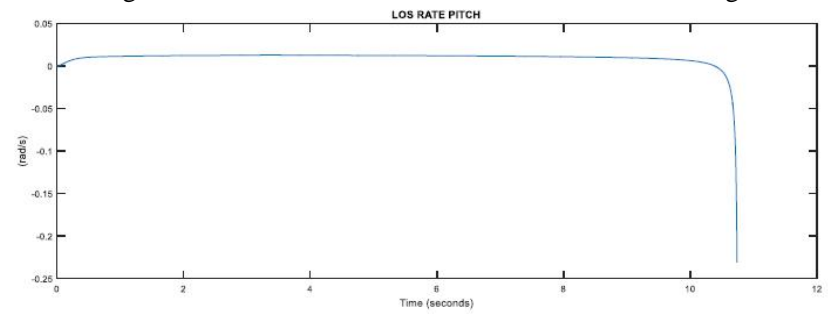

Fig. 15. LOS rate in Pitch Direction between Missile and Target

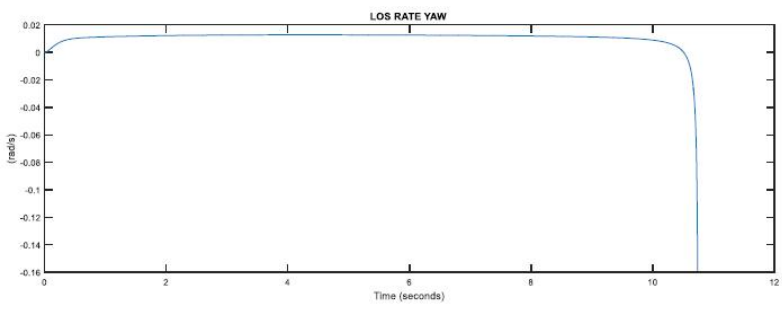

Fig. 16. LOS rate in Yaw Direction between Missile and Target

We already discussed about tracking controller, how control system can make the system follow the input exactly. It is very important for the missile to follow the direction of input exactly same so the guidance would not miss too much. From the simulation, we know that our system is powerful enough to follow the input as shown in Fig. 15 and Fig. 16.

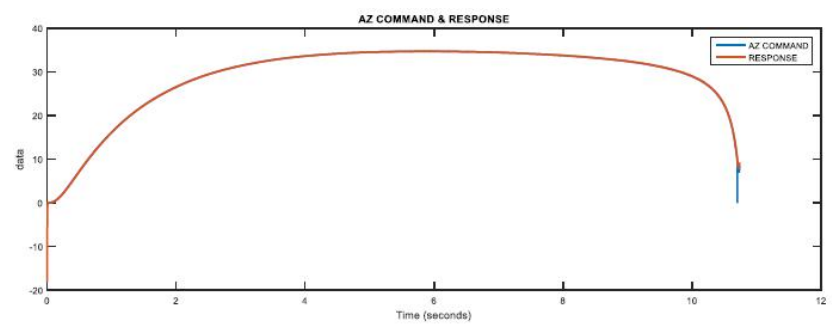

Fig. 17. AZ Command (Blue) and Response (Red)

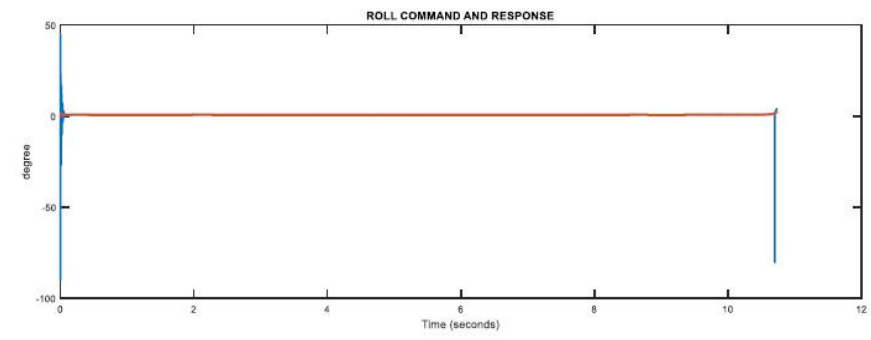

Fig. 18. Roll Angel Command (Blue) and Response (Red)

\section{CONCLUSION}

Tracking controller system has good approximation. This can be shown from the result that az and roll command is inline with response.

Design of guidance system using proportional navigation method can be implemented or used for preliminary design purpose. It is because miss distance of the simulation is less than 10 meter.

Proportional navigation method for AIM 120 AMRAAM has powerful performance. It can be shown from the loss rate of the missile along the trajectory is zero along the way to the target.

\section{REFERENCES}

[1] Blake, William. 2011. Missile Datcom User's Manual, United States : U.S. Army Aviation \& Missile Research, Development and Engineering Center.

[2] B. Friedland, D. E. Williams. 1989. Design of Autopilot for Bank-toTurn Missile. United States: Kearfott Guidance and Navigation Corporation.

[3] Francis C, Lukenhill. 1990. A Target/Missile Engagement Scenario using Classical Proportional Navigation, United States: Naval Postgraduate School. 
[4] Laksmana, Yayom D. 2016. Perancangan dan Simulasi Sistem Panduan, Sistem Kendali, serta Dinamika Terbang AIM 120 AMRAAM. Indonesia: Bandung Institute of Technology.

[5] Mulder J.A. et al. Flight Dynamics Lecture Notes. Belanda: TUDelft.

[6] Schmidt, David. Modern Flight Dynamics. 2012. New York: McGraw-Hill.

[7] http://militaryedge.org/armaments/aim-120-amraam/ (Accessed in August, $14^{\text {th }} 2017,7: 34$ PM.

\section{APPENDIXS A}

VARIABLE IN EQUATIONS MEANING

\begin{tabular}{ll} 
Parameter & \multicolumn{1}{c}{ Meaning } \\
& Acceleration of missile \\
& Acceleration of missile in pitch \\
& Acceleration of missile in yaw \\
& Seeker coefficient \\
& Seeker Coefficient \\
& Guidance Coefficient \\
& Subscript for missile \\
Vubscript for target & \\
Velocity & Position in x-axis; subscript \\
& for component in x-axis \\
& Position in y-axis; subscript \\
& for component in y-axis \\
& Position in z-axis; subscript \\
& for component in z-axis
\end{tabular}

Flight path angels

Line of Sight Angels 
